\title{
OPEN Designing a multi-epitope vaccine against Mycobacteroides abscessus by pangenome-reverse vaccinology
}

\author{
Hamza Arshad Dar ${ }^{1,5}$, Saba Ismail ${ }^{1,5}$, Yasir Waheed ${ }^{1 凶}$, Sajjad Ahmad ${ }^{1}$, Zubia Jamil ${ }^{1}$, \\ Hafsa Aziz ${ }^{2}$, Helal F. Hetta ${ }^{3} \&$ Khalid Muhammad ${ }^{4 凶}$
}

Mycobacteroides abscessus (Previously Mycobacterium abscessus) is an emerging microorganism of the newly defined genera Mycobacteroides that causes mainly skin and tissue diseases in humans. The recent availability of total 34 fully sequenced genomes of different strains belonging to this species has provided an opportunity to utilize this genomics data to gain novel insights and guide the development of specific antimicrobial therapies. In the present study, we collected collectively 34 complete genome sequences of $M$. abscessus from the NCBI GenBank database. Pangenome analysis was conducted on these genomes to understand the genetic diversity and to obtain proteins associated with its core genome. These core proteins were then subjected to various subtractive filters to identify potential antigenic targets that were subjected to multi-epitope vaccine design. Our analysis projected the open pangenome of $M$. abscessus containing 3443 core genes. After applying various stepwise filtration steps on the core proteins, a total of four potential antigenic targets were identified. Utilizing their constituent CD4 and CD8 T-cell epitopes, a multi-epitope based subunit vaccine was computationally designed. Sequence-based analysis as well as structural characterization revealed the immunological effectiveness of this designed vaccine. Further molecular docking, molecular dynamics simulation and binding free energy estimation with Toll-like receptor 2 indicated strong structural associations of the vaccine with the immune receptor. The promising results are encouraging and need to be validated by additional wet laboratory studies for confirmation.

Mycobacteroides abscessus (Previously called Mycobacterium abscessus) is an emerging microorganism that is increasingly becoming resistant to antibiotic therapy and causes diseases related to skin and soft tissues ${ }^{1,2}$. According to the revised bacterial nomenclature system, Mycobacteroides is the name of new genera (proposed in 2018) that was identified on the basis of 51 unique molecular markers and belongs to the Abscessus-Chelonae clade that generally exhibits fast growth ${ }^{3,4}$.

Mycobacteroides abscessus is a highly notorious and common member of the Mycobacteroides genus. $M$. abscessus commonly poses numerous therapeutic challenges on account of its innate resistance to majorly used antibiotics ${ }^{5}$. Some mortal cases in Cystic Fibrosis patients have also been reportedly linked to these infections . $^{6}$ The seriousness of this situation can be gauged by the fact that in many countries, lung transplantation is not recommended in case of $M$. abscessus infection thus rendering CF patients hopeless. The pathology of their infection is mostly related to a transition from a smooth " $\mathrm{S}$ " form to rough the " $\mathrm{R}$ " form". The emergence of this bacterial species has been largely attributed to the increased use of broad-spectrum antibiotic therapy.

The introduction of Next Generation Sequencing and other whole genome sequencing-based technologies has revolutionized the field of genomics ${ }^{8}$. This, along with recent advancements in the field of bioinformatics, has resulted in the accumulation of substantial genomics data of many microorganisms in publicly available biological databases. At the time of writing, a total of 34 genome sequences of $M$. abscessus were available in the GenBank. Therefore, we aimed to explore this data using bioinformatics tools to enhance our understanding of the genome scale analysis of this opportunistic microorganism and identify potential targets to guide vaccine discovery. This was important considering that conventional approaches in vaccinology take a lot of

\footnotetext{
${ }^{1}$ Foundation University Medical College, Foundation University Islamabad, DHA-I, Islamabad 44000, Pakistan. ${ }^{2}$ Nuclear Medicine, Oncology, and Radiotherapy Institute, Islamabad 44000, Pakistan. ${ }^{3}$ Department of Medical Microbiology and Immunology, Faculty of Medicine, Assiut University, Assiut 71515, Egypt. ${ }^{4}$ Department of Biology, College of Science, United Arab Emirates University, 15551 Al Ain, United Arab Emirates. ${ }^{5}$ These authors contributed equally: Hamza Arshad Dar and Saba Ismail. ${ }^{\bowtie}$ email: yasir.waheed@fui.edu.pk; k.muhammad@ vaeu.ac.ae
} 
time, resources and expertise ${ }^{9}$. Briefly, these studies involve complicated steps like microbial culturing, isolation of antigen, inactivation and reinjection of these antigens into subjects to verify the immunogenic potential. Furthermore, these classical approaches are not feasible to conduct large scale analysis on a variety of bacterial isolates/strains. In such situations, there is a need to identify conserved antigenic target, prepare it, and evaluate its utility for immunization applications.

This study attempted to address the therapeutic challenges arising due to the worsening antimicrobial resistance within M. abscessus. For this purpose, complete genome sequences of this opportunistic bacterial pathogen were obtained from the NCBI GenBank database. An integrative analysis was thus conducted encompassing pangenome analysis, reverse vaccinology, immunoinformatics as well as structural bioinformatics to identify promising targets to design a universally applicable chimeric vaccine. This was especially relevant considering the lack of such studies in the literature concerning an emerging microorganism of public health interest.

We believe that the putative vaccine targets fulfilled the criteria to act as a source of epitopes for developing a polyepitope vaccine. Further the three-dimensional structure prediction was performed on the multi-epitope vaccine. Molecular docking analysis with Toll-like receptor 2 was also conducted to evaluate the vaccine's effectiveness. Altogether, we believe that this study will provide updated knowledge about the global gene repertoire of an emerging bacterial species and guide novel vaccine design to counter these infections.

\section{Material and methods}

Collection of genomics data. The genomics and proteomics data of total 34 complete M. abscessus genomes was collected from the GenBank database of NCBI. Additional information, such as strain name, GC\%, accession number, and number of contigs, was also collected.

Pangenome analysis. Pangenome analysis was carried out on the 34 genomes using the BPGA, a quick genome analysis pipeline ${ }^{10}$. For clustering sequences, a $90 \%$ sequence identity threshold was specified in USEARCH algorithm ${ }^{11}$. BPGA standalone tool computes pan and core genome size by 20 permutations and stating median values after the inclusion of every genome. The core and pan genome graphs are obtained by analyzing the total number of common and unique gene families against the total number of genomes, respectively. Additionally, BPGA can generate a gene family distribution plots illustrating core pan and accessory genes. The output also yields a distribution plot of new genes which shows how many new genes are added with the inclusion of each successive genome. The protein sequences associated with the $M$. abscessus core genome were also obtained and subjected to additional filters to identify potential therapeutic targets.

Reverse vaccinology approach to select Potential Vaccine Candidates. To guide the selection of potential core vaccine targets, Vaxign server (http://www.violinet.org/vaxign/) was used. Vaxign is the first reverse vaccinology tool to identify suitable antigenic targets in bacterial genomes (mostly) ${ }^{12}$. For this purpose, "Dynamic Analysis" mode was used to query the core genome-related proteins.

The first inclusion criteria applied to the core genome proteins were subcellular localization restricted to the cell wall or extracellular regions. Exoproteome and secreted substances associated with bacteria are considered to be useful antigenically as they are exposed outside the bacterial cell and can easily develop interactions with circulating immune cells ${ }^{13}$. For cross-validation of subcellular localization, two popular bioinformatics servers were used: PSORTb v3.0.2 (available at http://www.psort.org/psortb/) and CELLO v.2.5 (available at http://cello. life.nctu.edu.tw/).

The exclusion criteria applied to the non-homologous proteins was homology to humans and related organisms (mice and pig). Due to significant similarity with the human proteins, either autoimmunity or tolerance to foreign antigens could occur ${ }^{14}$. This can have harmful consequences for human health. Therefore, bacterial proteins found to exhibit homology to host protein(s) are generally not good candidates for developing a safe vaccine.

The adhesion potential of targets was assessed using Vaxign. The threshold for adhesion selection was set to 0.5 . Proteins having adhesive properties interact with the host cellular receptors and facilitate bacterial attachment ${ }^{15}$. Specific antibodies can thus be generated against these foreign proteins by the host and may serve to activate protective immunity ${ }^{16}$. Thus, we took an aim to explore this aspect.

The final prioritization step by Vaxign was the number of transmembrane helices less than two. This is usually suggested to ensure that purification of these targets is done in an appropriate wet laboratory setting ${ }^{9}$.

Next, the antigenic tendency of the predicted proteins was analyzed using the VaxiJen 2.0 server ${ }^{17}$. Score cutoff was adjusted to 0.5 . The good thing about this server is the lack of dependence on alignments. With accuracy within $70-89 \%$ range, this tool is known to be very accurate and may lead to fewer false positive hits, which is highly desirable in the reverse vaccinology approach.

The Protparam tool was used to compute the physicochemical features of selected proteins ${ }^{18}$. In particular, four things were assessed. Firstly, molecular weights were analyzed to verify whether they are of low weight (less than $110 \mathrm{kD})^{19}$. Secondly, the Instability Index was checked to confirm stability. Finally, the hydrophobicity and aliphatic index were scrutinized to select hydrophilic molecules with good thermal stability. The negative GRAVY index provided an indication of hydrophilicity whereas a higher aliphatic index $(>50)$ suggested high stability at varying temperature ${ }^{20}$.

Prediction of CD8 T cell interacting epitopes. HLA class I epitope prediction was done through VaxiTop analysis through Vaxign server ${ }^{12}$. Default $P$ value $(0.05)$ was selected, the alleles specified were HLA-A0101, HLA-A0201, HLA-A0301, HLA-A1101 and HLA-B3501, organism selected was human, and only epitopes lying outside cell were considered due to their maximum exposure to the immune cells outside the cell. The predicted epitopes were further scrutinized on the basis of their immunogenicity scores calculated by the immunogenicity 
tool of the IEDB ${ }^{21,22}$. The antigenicity filter was also applied on the proteins by the VaxiJen 2.0 server, with 0.8 cut-off score ${ }^{17}$. Finally, the allergenicity of the 9-mer peptide sequences was evaluated using the AllergenFP 1.0 tool to remove potential allergens ${ }^{23}$.

Prediction of CD4 T cell interacting epitopes. To predict T-cell epitopes having the tendency to interact with HLA class II, NetMHCIIpan 3.2 server (available at http://www.cbs.dtu.dk/services/NetMHCIIpan-3.2/) was used (Jensen et al., 2018). Default threshold for categorizing binding affinity level to shortlist strong binders. DRB alleles specified were DRB1_0101, DRB1_0701, DRB1_0301, DRB1_0401, DRB1_0801, DRB1_1101, DRB1_1501 and DRB1_1301. The predicted results were sorted as per the binding affinity values. The antigenicity screening of top binders for each allele were conducted using the VaxiJen server ${ }^{17}$. Only those epitopes scoring above 0.5 threshold were selected. Wherever there were overlapping peptides, those having the most antigenic values were retained for that allele.

In silico design of multi-epitope vaccine and structural modeling. The shortlisted epitopes were attached to each other using a specialized Glycine-Proline linker sequence of GPGPG. Towards the N-terminal direction of the epitopes, Cholera Toxin subunit B, a molecular adjuvant was incorporated. This adjuvant was connected to the epitopes via one EAAAK rigid linker. The physical and chemical properties of vaccine such as theoretical $\mathrm{pH}$, half-life, molecular weight, grand average of hydropathicity, aliphatic and instability indexes, were computationally analyzed using the Expasy tool of Protparam ${ }^{18}$. Furthermore, the designed multi-epitope vaccine was subjected to further antigenicity and allergenicity checks, using VaxiJen and AllergenFP servers, respectively ${ }^{17,23}$.

After sequence-based analysis, the three-dimensional structure prediction was performed. For this purpose, the 3DPro tool was utilized ${ }^{24}$. The crude model obtained was then subjected to further improvements via Galaxyrefine 2 server through simulations to enhance its structural quality ${ }^{25}$. This was corroborated through different tools like ProSA-web and PROCHECK to ensure the availability of a structurally refined and stable multi-epitope vaccine for the next steps ${ }^{26,27}$.

In silico C-immune simulation. The designed construct was subjected to C-ImmSim server for determination of its in silico immunogenic potential ${ }^{28}$. The server operates by application of machine learning techniques and position-specific scoring matrix (PSSM) for evaluation of host immune response towards the antigen.

Vaccine-TLR molecular docking analysis. The binding affinity of the vaccine to TLR2 was explored using a molecular docking technique to validate the vaccine's immunological effectiveness. M. abscessus infection leads to TLR2-mediated immune responses in humans ${ }^{29}$. The structure of TLR2 was obtained from a crystal structure with PDB ID $2 Z_{7 X}^{30}$.

The protein structural files PDB were uploaded to the HADDOCK system guru interface using default options $^{31}$. To obtain active and passive residues, the CPORT server was used ${ }^{32}$. Docking clusters were obtained and one cluster with the minimum HADDOCK score was uncovered. From this cluster, one docked PDB file was proceeded towards the refinement stage to improve the orientation of biomolecules towards one another. The UCSF Chimera tool ${ }^{33}$ and $\mathrm{VMD}^{34}$ visualized the docked vaccine-TLR structure. To identify and highlight the crucial binding interface within the docked complex, PDBsum server was used ${ }^{35}$.

Binding affinity analysis of vaccine-TLR2 complex. The vaccine-TLR2 complex was submitted to the PRODIGY server (available at http://milou.science.uu.nl/services/PRODIGY) to predict the vaccine-TLR2 binding strength ${ }^{36}$. The prediction is carried out by exploiting information such as intermolecular contacts as well as non-interface properties. Default parameters were selected to conduct this analysis.

Molecular dynamics simulation. The docked complex of vaccine-TLR2 was subjected to a 50 ns production run of molecular dynamics simulation to understand the system dynamics in aqueous solution. Molecular dynamics simulation was performed using AMBER18 software ${ }^{37}$. Both TLR2 and vaccine parameters were prepared using the ff14SB force field ${ }^{38}$. Complex integration into a TIP3P water box was accomplished in the process where the padding distance was set to $12 \AA$. Neutralization of the system was done by adding $\mathrm{Na}+$ ions. System hydrogen atoms, solvation box, carbon alpha atoms, and all non-heavy atoms were minimized for 500, 1000,1000 , and 300 steps, respectively. Subsequently, heating of the system to $300 \mathrm{~K}$ for 20 -ps was done via Langevin dynamics to maintain the system temperature ${ }^{39}$. Here, the restraint of $5 \mathrm{kcal} / \mathrm{mol}-\mathrm{A} 2$ on carbon alpha atoms at time step of 2 fs was allowed. In equilibration, system was relaxed for 100 ps. System pressure was maintained by means of NPT ensemble for 50-ps. Lastly, a production run of $50 \mathrm{~ns}$ was accomplished at the time interval of $2 \mathrm{fs}$. Generated trajectories were analyzed for structural parameters using AMBER CPPTRAJ ${ }^{40}$. The hydrogen bonds formed throughout the trajectories between TLR2 and vaccine were plotted in VMD ${ }^{34}$, setting an angle of $30^{\circ}$ and a bond distance of $0.35 \mathrm{~nm}$.

MM-PB/GBSA studies. Intermolecular binding free energies of the system were estimated using MMPBSA. py package of AMBER18 program ${ }^{41}$. The overall objective of this analysis was to evaluate the free energy difference between two states (solvated and gas phase) of the complex. The net binding free energy was calculated as, 


\section{Core-Pan Plot}

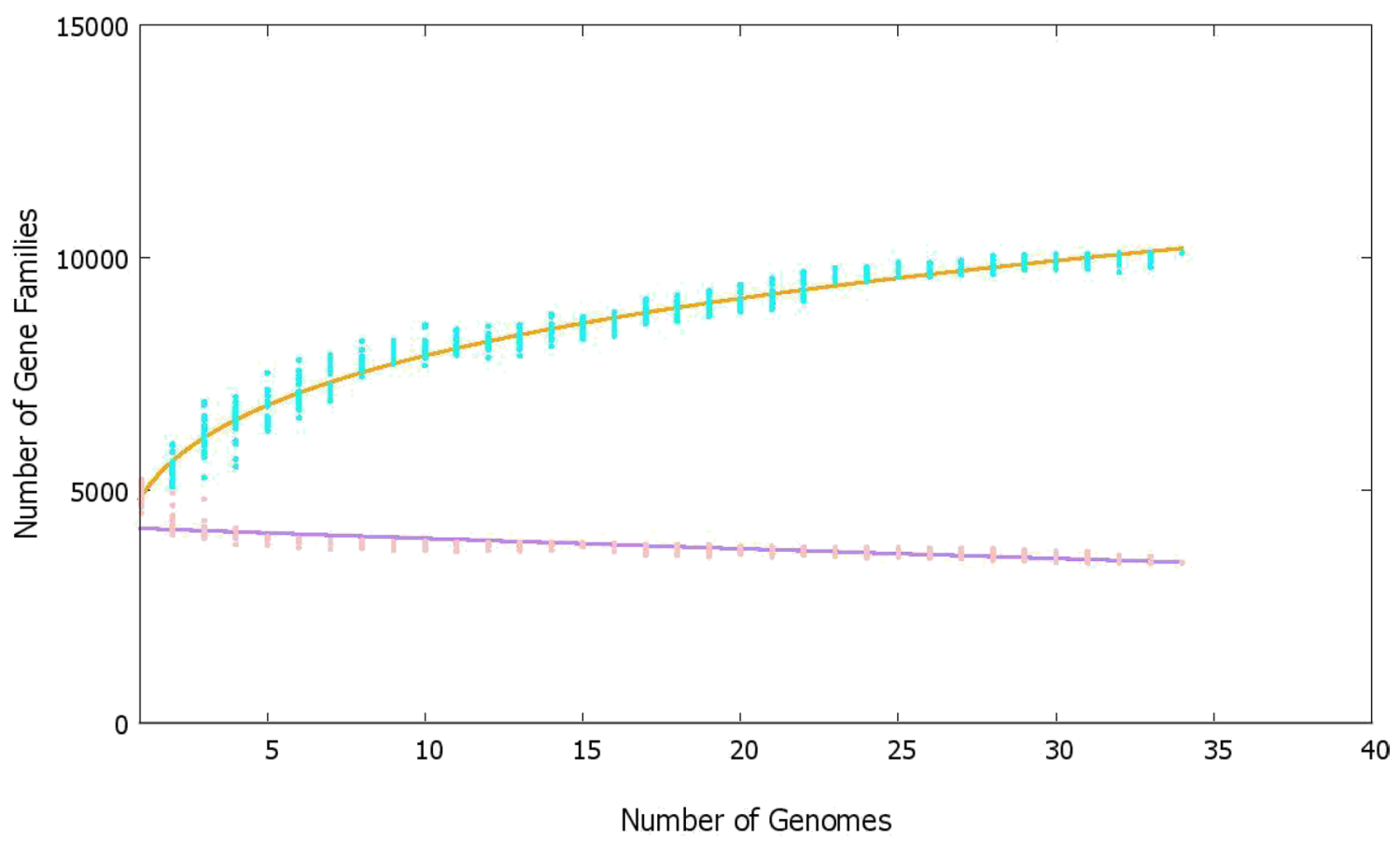

Power-fit Curve Equation: $\mathrm{f}(\mathrm{x})=4877.12 \cdot \mathrm{x}^{0.21}-$ Exponential Curve Equation: $f 1(x)=4208.02 \cdot e^{-0.01} \cdot x$ Core gene families

Figure 1. Pan-core plot of 34 Mycobacteroides abscessus genomes. With the inclusion of each new genome, the size of the pangenome increased while that of the core genome decreased. The pangenome curve (shown in brown) continues to show an upward trajectory. This suggests that the global gene pool of M. abscessus is likely to increase in the future.

$$
\begin{aligned}
& \Delta G \text { binding free energy }=\Delta G \text { bind, vaccum }+\Delta G \text { solv, complex } \\
& \qquad-(\Delta G \text { solv, ligand })+\Delta G \text { solv, receptor } \\
& \qquad \begin{aligned}
\Delta G \text { solv }= & \Delta G \text { electrostatic, } \varepsilon=80+\Delta G \text { electrostatic, } \varepsilon \\
= & 1+\Delta G \text { hydrophobic }
\end{aligned}
\end{aligned}
$$

$\Delta G$ vaccum $=\Delta E$ molecular, mechanics $-T \cdot \Delta G$ normal $\bmod e$ analysis

In total, 100 frames were extracted from simulation trajectories to be used in both MMGBSA (Molecular Mechanics Generalized Born Surface Area) and MMPBSA (Molecular Mechanics Poisson-Boltzmann Surface Area).

\section{Results}

Pangenome analysis and retrieval of the core proteome. 34 complete genomes of M. abscessus were obtained from GenBank. The information of these strains such as strain name, accession number, as well as genome statistics are provided in Supplementary file 1. It was found through pangenome analysis of M. abscessus strains that its core genome size is 3443 . Meanwhile, the total number of gene families (pangenome) was calculated to be 10,110 . The core/pangenome size ratio was found to be 0.340 , thus the core forms $34 \%$ of the pangenome. This signifies the high level of genetic diversity in the M. abscessus species. The pan-core plot (Fig. 1) also visualized this trend. The expansion rate 'b' was found to be 0.2093 which indicates that the pangenome of this species is open and has the potential to increase. Each genome on average contained a total of 4893 proteinencoding genes. The core genome size accounted on average for $70 \%$ of average genome size. The smallest number of protein-encoding genes were present in GO 06 strain i.e., 45,212; Meanwhile, strain FLAC055 contained a total of 5237 genes. The pangenome and core genome phylogenetic trees were constructed and subsequently visualized (Figs. 2 and 3). 


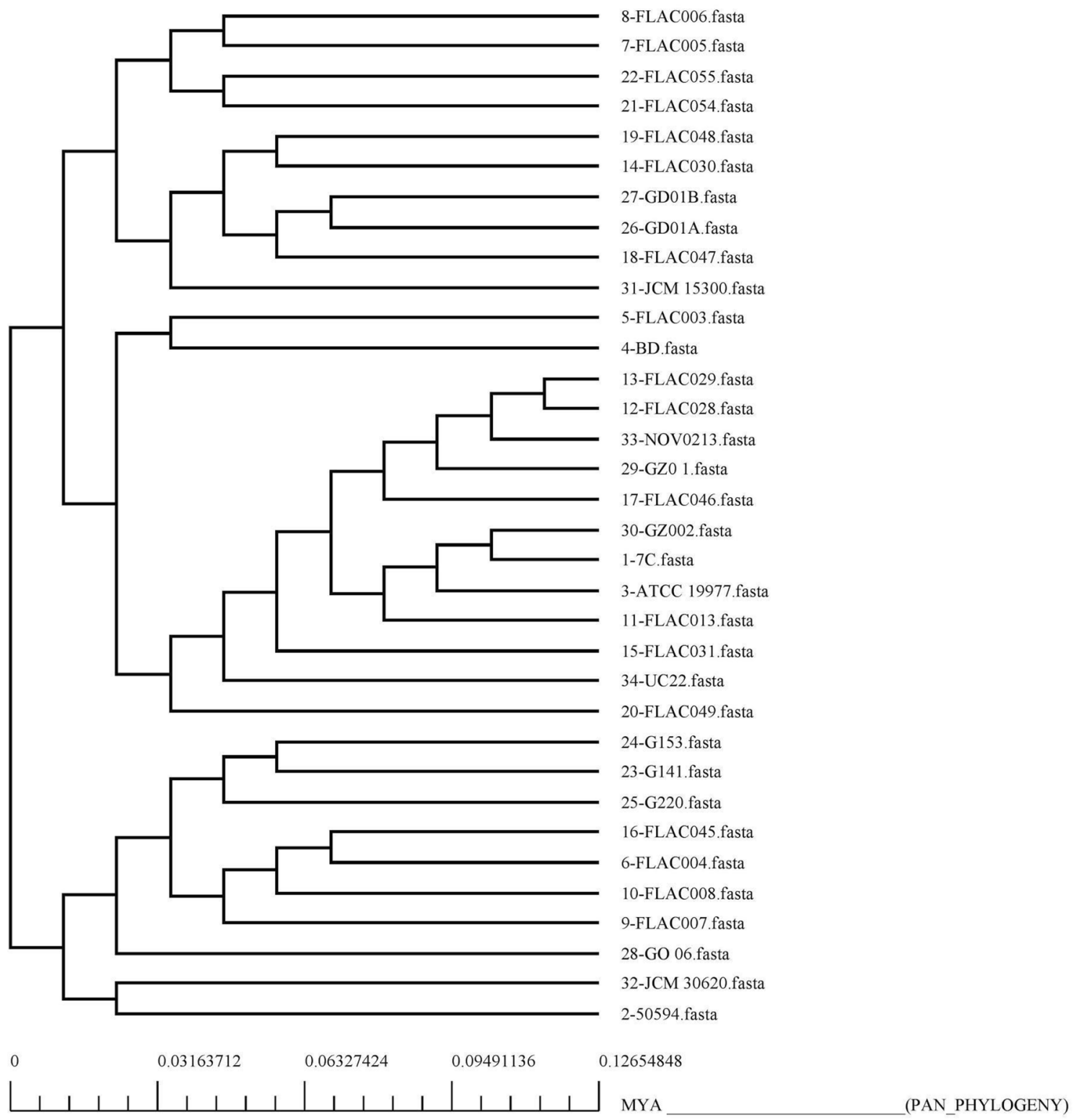

Figure 2. Panphylogeny tree of 34 complete genomes of Mycobacteroides abscessus. This pan-phylogeny has been constructed on the basis of accessory gene presence/absence data in different strains.

Identification of potential core vaccine candidates in Mycobacteroides abscessus. The analysis of the core genome-associated proteins through Vaxign revealed that out of 4443 proteins, only 45 had subcellular localization restricted/confined to the extracellular or cell wall regions (Supplementary file 2).

The homology analysis of these confirmed that the total six targets were homologs of humans, mice and/or pigs, hence they were removed from further consideration to retain 39 proteins (Supplementary file 3). Next, the adhesion filter was applied, and it was found that a total of 19 proteins had putative adhesion properties (Supplementary file 4). All of them had less than two transmembrane helices.

After Vaxign, additional filters were applied. The antigenicity evaluation of the targets revealed that the total 10 targets had antigenic tendencies and thus were good vaccine candidates (Supplementary file 5). It was found through physicochemical analysis that out of these 10 proteins, only four exhibited favorable properties such as low molecular weight, high aliphatic index and instability index less than 40 as well as negative value of the GRAVY index (Supplementary file 6). These observations further support the immunological relevance of our polyepitope vaccine.

Subcellular localization cross-check was performed on the 45 proteins predicted as extracellular or cell walllocalized by Vaxign for validation. PSORTb v3.0.2 revealed that 37 proteins were extracellular while the remaining eight were cell wall-localized targets (Supplementary file 7). Whereas, according to CELLO server, 24 out of 45 proteins were classified as extracellular and the remaining 21 were regarded as cytoplasmic/membrane or multiple-localized targets (Supplementary file 7). However, even by retaining this strict cross-verification 


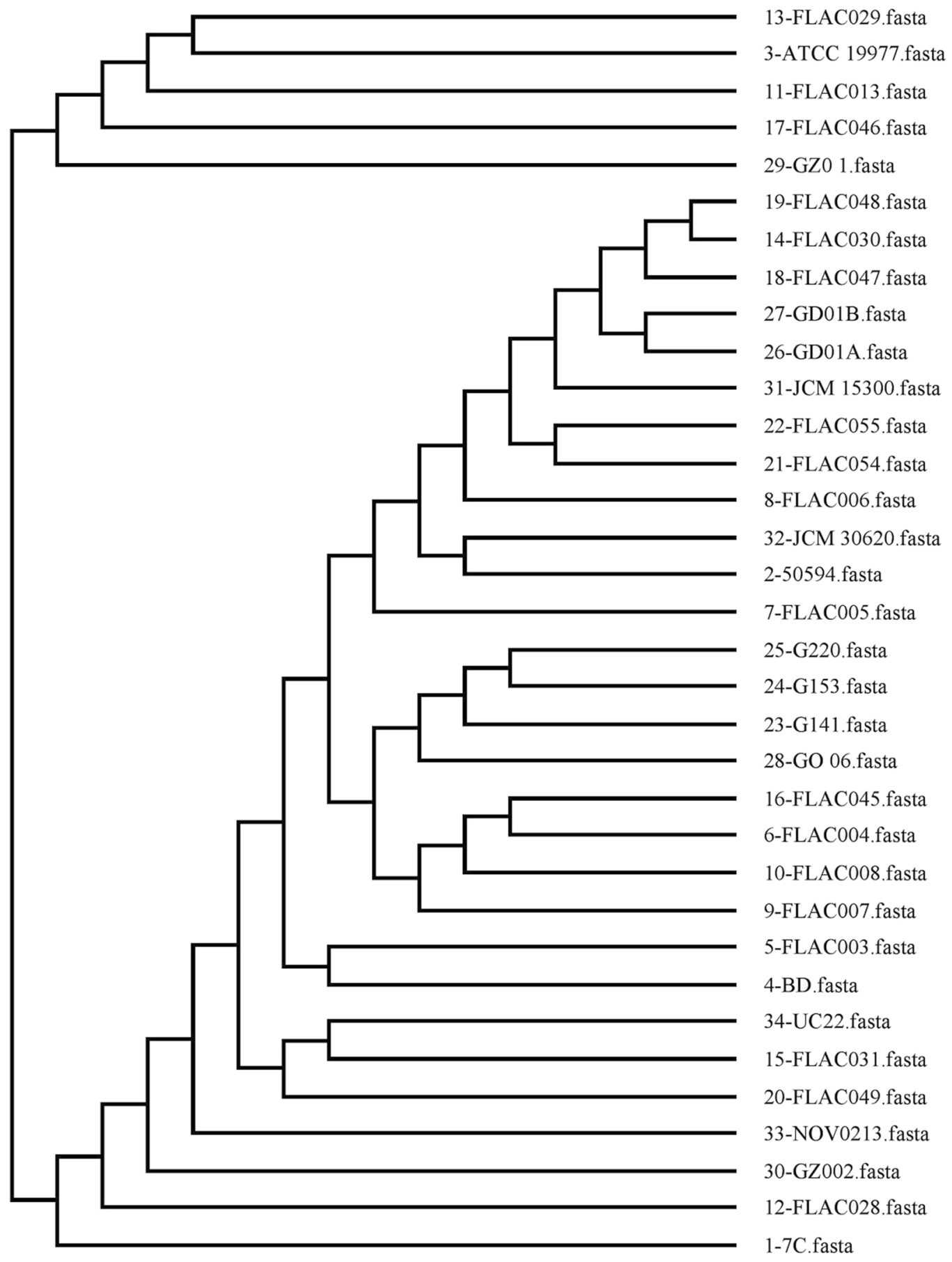

0

$0.0024357257327250 .00487145146545 \quad 0.0073071771981750 .0097429029309$

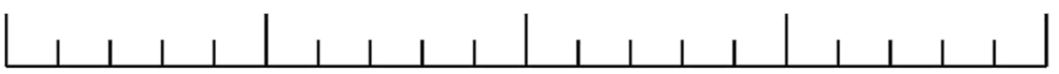

MYA (CORE PHYLOGENY)

Figure 3. Core genome tree of 34 complete genomes of Mycobacteroides abscessus. This core phylogeny has been obtained on the basis of concatenated alignment of proteins associated with the core genome.

standard, further prioritization steps of 24 extracellular proteins yielded the same four antigen targets obtained in our study. Also, all four antigens were classified as extracellular by both PSORTb v3.0.2 and CELLO v 2.5. So, as a whole, this analysis provided verification of subcellular localization through multiple credible tools.

BLAST analysis of these proteins revealed that two out of these were hypothetical proteins so their functional annotation is not available. Whereas the remaining two are Resuscitation-promoting factor (RPF) and invasion protein Inv1. RPF is present in numerous gram positive bacteria responsible for reactivating cultures from stationary phase. RPF family is very likely to act as trans glycosylase enzymes similar to PF00062 and PF01464 


\begin{tabular}{|l|l|l|l|l|l|l|}
\hline Model & RMSD & Mol probity & Clash score & Poor rotamers & Rama favored & GALAXY energy \\
\hline Initial & 0.000 & 4.145 & 180.6 & 6.5 & 73.2 & $52,633.20$ \\
\hline MODEL 1 & 2.505 & 1.461 & 2.2 & 0.6 & 92.4 & -4411.11 \\
\hline MODEL 2 & 2.110 & 1.423 & 1.9 & 0.0 & 92.4 & -4398.08 \\
\hline MODEL 3 & 2.068 & 1.104 & 0.3 & 0.0 & 91.5 & -4396.93 \\
\hline MODEL 4 & 1.876 & 1.420 & 1.4 & 0.0 & 89.7 & -4379.94 \\
\hline MODEL 5 & 2.229 & 1.527 & 2.4 & 0.6 & 91.5 & -4369.58 \\
\hline MODEL 6 & 2.249 & 1.343 & 1.1 & 0.0 & 90.6 & -4368.67 \\
\hline MODEL 7 & 2.006 & 1.569 & 2.4 & 0.0 & 90.2 & -4364.43 \\
\hline MODEL 8 & 2.246 & 1.521 & 2.2 & 0.0 & 90.6 & -4361.25 \\
\hline MODEL 9 & 2.227 & 1.520 & 1.9 & 0.6 & 89.3 & -4359.12 \\
\hline MODEL 10 & 1.904 & 1.408 & 1.4 & 0.0 & 90.2 & -4350.07 \\
\hline
\end{tabular}

Table 1. Information about structural properties of the ten models generated after refining the structure of the crude multi-epitope vaccine.

(http://academic.oup.com/femspd/article/58/1/39/557188). This protein also shares structural elements with lysozyme and associated proteins. Inv exact function is not known however it bears similarity to peptidoglycan endopeptidase RipA of Mycobacterium tuberculosis variant bovis BCG and cleaves the bond between D-glutamate and meso-diaminopimelate according to Uniprot (https://www.uniprot.org/).

Identification of potential HLA class I epitopes in PVCs. Analyzing the sequence of four core antigenic proteins using the VaxiTop resulted in the identification of 82 HLA I T-cell epitopes (Supplementary file 8). Out of these, a total of 44 epitopes were positively selected on account of their immunogenicity as reflected by the positive immunogenicity scores by the IEDB server (Supplementary file 9). Further scrutinization of antigenicity and non-allergenicity prioritized four HLA I T-cell epitopes. These are QADETNATM, RVAENVLAR, LTATNPDDV and YYGGLQFNL.

Identification of potential HLA class II epitopes in PVCs. Analysis through NetMHCIIpan3.2 server revealed the presence of total 23 HLA class II epitopes within the four core antigenic proteins (Supplementary file 10). After antigenicity screening and removal of overlapping epitopes, a total of four epitopes were retained. These are LWAMAPALVAAPMAL, LLIFAFLGVTAAVGV, GGDLELFKNATATMK, and SREEQIRVAENVLAR.

The in silico design of polyepitope vaccine and sequence-based evaluation. The prioritized epitopes were linked together and with Cholera Toxin B to envision a multi-epitope subunit vaccine. Since HLA I T-cell epitope RVAENVLAR was found to lie within the prioritized HLA II T-cell epitope SREEQIRVAENVLAR, so out of these two, only the later epitope was incorporated. The polyepitope vaccine is comprised of 226 number of amino acids. Molecular weight of the recombinant antigen was found to be 23,657.02 Daltons. Isoelectric $\mathrm{pH}$ value is 5.81 . The half-life is $30 \mathrm{~h}$ (mammalian reticulocytes, in vitro), at least $20 \mathrm{~h}$ (yeast, in vivo) and at least $10 \mathrm{~h}$ (Escherichia coli, in vivo). Whereas, its instability index was found to be $\sim 30$. If the instability index value of a given protein is less than 40 , the protein is deemed to be stable. A significantly high aliphatic index of 79.16 was computed which projects a considerable level of thermostability. Finally, the GRAVY value was calculated, and it was revealed that this designed vaccine has a negative value of GRAVY index $(-0.143)$. This means that the vaccine is predicted to have a hydrophilic character and thus likely to engage closely with water, a desirable attribute of vaccine. The sequence of the polyepitope vaccine is provided below in plain format:

MTPQNITDLCAEYHNTQIHTLNDKIFSYTESLAGKREMAIITFKNGATFQVEVPGSQHIDSQKKAIERMKDTLRIAYLTEAKVEKLCVWNNKTPHAIAAISMANEAAAKQADETNATMGPGPGLTATNPDDVGPGPGYYGGLQFNLGPGPGSREEQIRVAENVLARGPGPGGGDLELFKNATATMKGPGPGLWAMAPALVAAPMALGPGPGLLIFAFLGVTAAVGV.

Both AllergenFP and AllerTOP 2.0 servers projected the non-allergenicity of multi-epitope vaccine. According to the VaxiJen server results, the antigenicity of the vaccine is 0.7973 i.e. above the threshold of 0.5 and thus highly antigenic. After sequence-based characterization, we proceeded towards structural analysis of the vaccine.

Structural modeling of polyepitope-based subunit vaccine. Utilizing the intensive mode of the Phyre 2 server, we were able to model a total of 142 residues (63\%) with greater than $90 \%$ accuracy. However, critical assessment of this structure revealed that it was still in crude form and needed to be improved. Molecular refinements applied to this crude model yielded a total of ten models (Table 1). Out of these, the top three models according to GALAXY energy score were assessed further. Considering the Ramachandran plot analysis, ProSAweb and GALAXY energy results, MODEL1 was ultimately selected (Fig. 4).

Conformational B-cell epitope mapping in designed subunit vaccine. The three-dimensional structure of multi-epitope vaccine was subjected to discontinuous (conformational) B-cell epitopes prediction. A total of 126 epitopes were projected by the use of Ellipro server (Supplementary file 11). Whereas, DISCO- 

PGLLIFAFLGVTAAVGV
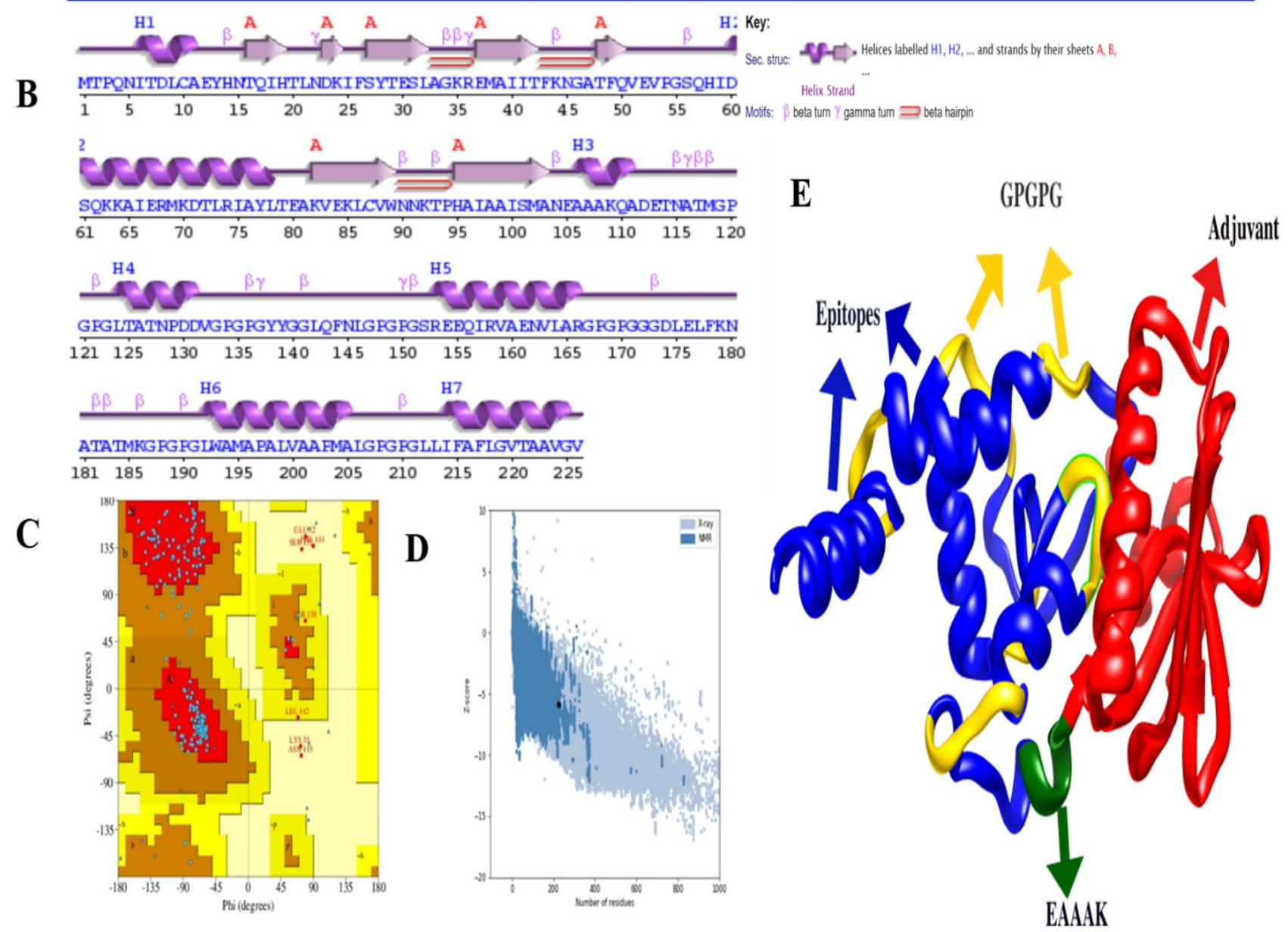

Figure 4. Structural characterization of multi-epitope vaccine construct: Polyepitope vaccine construct Sequence (A), Secondary Structure of polyepitope vaccine construct (B), PROCHECK graph of selected model (C), ProSA-web results showing Z-score of -5.83(D), Cartoon visualization of the designed vaccine 3D structure (E).

TOPE server collectively predicted 33 B-cell epitopes (Supplementary file 12). Figure 5A shows the epitopes predicted by Ellipro, whereas Fig. 5B visualizes the epitopes predicted by DISCOTOPE.

Population coverage of predicted T cell epitopes. In nature, MHC molecules are abundant and well distributed among various communities and in multiple ethnic groups. Therefore, the production of peptidebased vaccines, given most of the alleles, is a successful approach to broad-spectrum vaccine production. The $\mathrm{T}$ cell epitopes found in this study showed an average population coverage of $98.55 \%$ (MHC Class I), $81.81 \%$ (MHC Class II) and $99.74 \%$ (Combined). Figure 6 shows the known class I, class II and combined HLA alleles and the percentage of epitope group coverage.

In silico cloning of polyepitope based vaccine construct. The basic aim of in silico cloning of designed polyepitope based vaccine construct was to lead molecular biologists and genetic engineers on potential cloning sites and predict the level of expression in a particular expression system, for example in current study we used E. coli (K12) system. To produce its maximum expression, reverse translation of the designed polyepitope based vaccine construct's primary sequence was performed followed by cloning (Fig. 7). The CAI value of the enhanced construct sequence is 1, suggesting the vaccine's ideal expression. Whereas the GC content is $50.6 \%$ almost to the $E$. Coli $\mathrm{K} 12$, within the acceptable ranged from 30 to $70 \%$. 


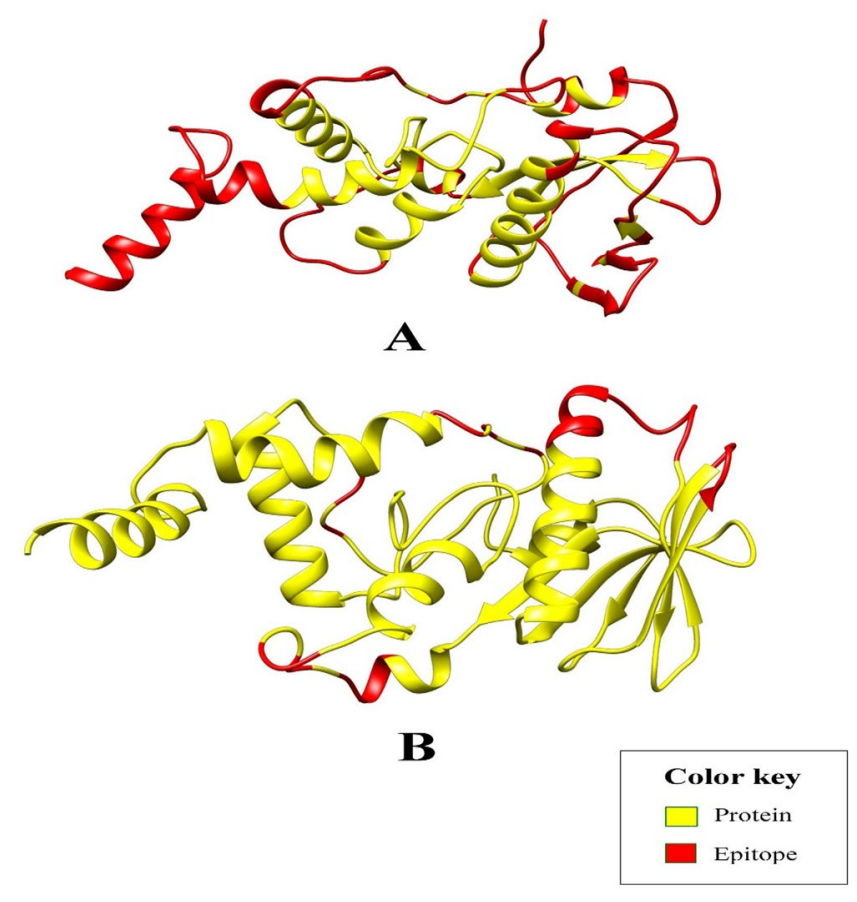

Figure 5. The discontinuous B-cell epitopes (red) within the multi-epitope vaccine as predicted by (A) Ellipro tool, and (B) DISCOTOPE server.

In silico immune simulation of polyepitope based designed vaccine construct. It appears that both primary and secondary immune responses play a crucial role against the pathogen and considered compatible with the real immune response. Figure 8 depicts the in silico based host immune response towards antigen. The primary response was characterized by high concentrations of $\operatorname{IgG}+\operatorname{IgG}$ and IgM, accompanied by primary and secondary concentrations of IgM, IgG1 + IgG2 and IgG1 with concomitant antigen reduction. In addition, strong interleukin and cytokine responses have been observed. All of this indicates the host's successful immune response and removal of pathogens upon successive encounters. In response to the antigen, the elevated B cell population, including memory cells and multiple isotypes, points to long-lasting memory development and isotype flipping. In addition to the cytotoxic $\mathrm{T}$ cell, the $\mathrm{T}$ helper cell population and their respective memory growth strongly agree with the strong response. In Fig. 9, the different population and per state count of immune cells are given.

Molecular docking revealed significant vaccine-TLR2 interaction. Molecular docking was carried out between our designed polyepitope-based vaccine and TLR-2 structures. To obtain the active as well as passive residues potentially used for establishing interactions, the CPORT server was used, and yielded information about these residues for each protein (Supplementary file 13). For molecular docking analysis, the advanced HADDOCK guru level system was utilized, with parameters set to default. CPORT-predicted active and passive residues were entered accordingly. Docking led to the clustering of the total 65 structures into 12 groups, which collectively represented $32.5 \%$ of the HADDOCK models obtained. The different statistical parameters of each cluster were analyzed and the top cluster having the lowest HADDOCK score $(-16.9 \pm 16.9)$ was identified. Docked structures of this cluster were visualized through chimera and VMD and one representative docked complex was then proceeded towards additional refinements.

Molecular refinements on the docked complex led to the placement of 20 clusters into one cluster which in this case represented $100 \%$ of HADDOCK models. This top cluster was observed to have a minimum HADDOCK score of $-164.4 \pm 2.6$. A docked structure from this cluster was visualized and interactive residues were noted accordingly (Fig. 10). Moreover, PDBsum analysis indicated that a total of 35 residues of the vaccine (with interface area $1616 \AA^{2}$ ) developed positive interactions with a total of 24 residues of the TLR2 (with interface area $1652 \AA^{2}$ ) (Fig. 10B). Some notable inter-chain hydrogen bonds were noted and visualized accordingly (Fig. 10C).

Binding affinity of vaccine-TLR2 complex. Utilizing the PRODIGY server, Gibbs free energy $(\Delta \mathrm{G})$ was obtained which is a crucial parameter to ascertain whether the vaccine-TLR2 complex formed was thermodynamically possible or not. It was found that $\Delta \mathrm{G}$ value is $-10.2 \mathrm{kcal} \mathrm{mol}^{-1}$ whereas the dissociation constant $\left(\mathrm{K}_{\mathrm{d}}\right)$ at $25^{\circ} \mathrm{C}$ was calculated to be $3.3 \mathrm{E}-08$. Therefore, this analysis confirmed that the vaccine-TLR complex formed is energetically feasible.

Molecular dynamics simulation studies. The dynamics and stable conformation of the docked vaccine molecule to TLR2 receptor were probed through a $50 \mathrm{~ns}$ run of molecular dynamics simulation. The output 

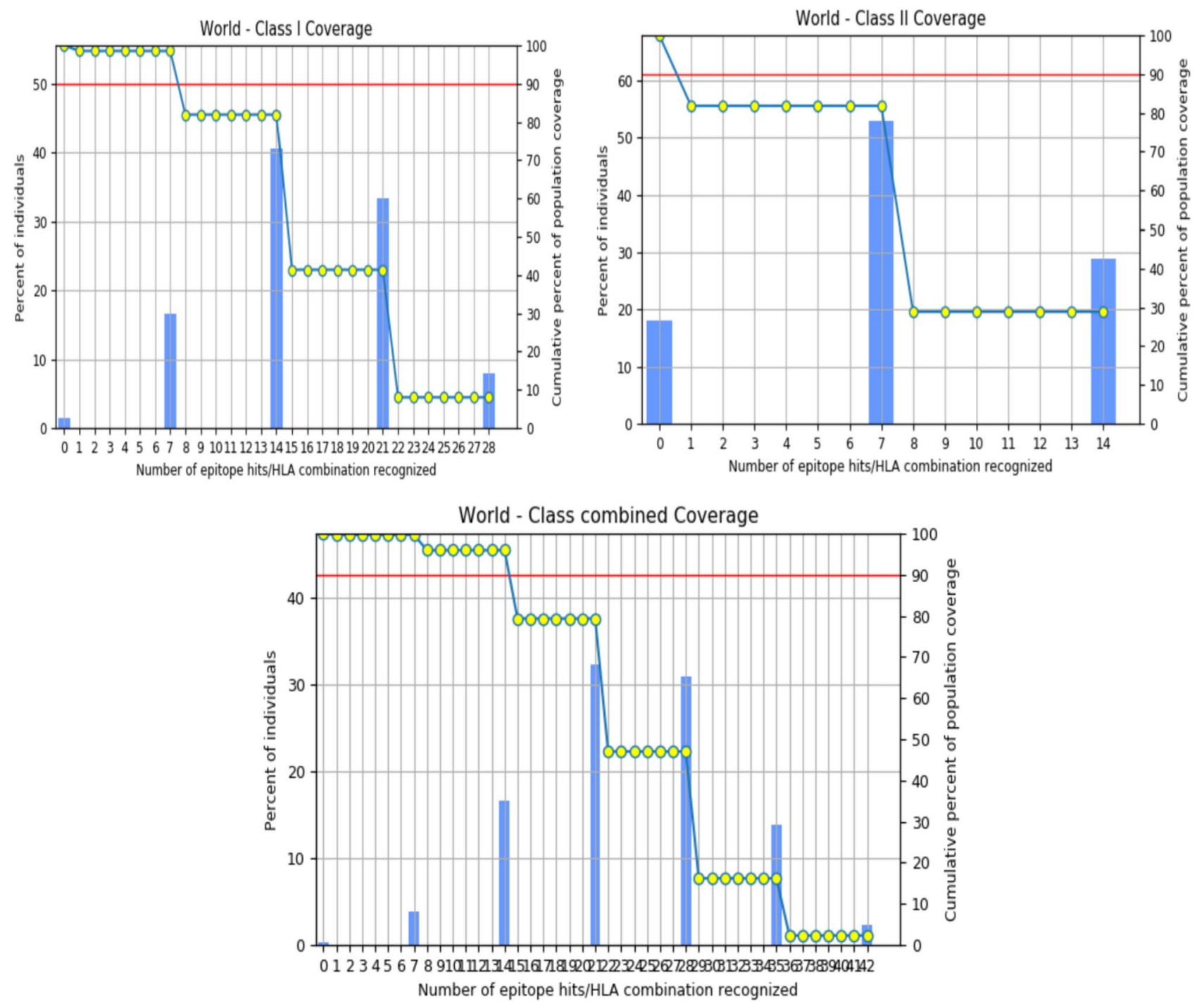

Figure 6. World class I, class II and combined coverage of HLA alleles recognized a set of predicted T cell epitopes.

trajectories were analyzed through four statistical parameters: Root mean square deviations (RMSD), Radius of gyration $\left(\mathrm{R}_{\mathrm{g}}\right)$, Root Mean Square Fluctuations (RMSF) and hydrogen bond analysis. All four parameters are illustrated in Fig. 11. RMSD was calculated for the TLR2 Ca atoms to decipher the structure stability among the superimposed snapshots obtained through simulation. The mean RMSD noticed for the system is $9.9 \AA$ (maximum of $15.9 \AA$ observed at frame 4558 ( $45.5 \mathrm{~ns})$. Generally, the RMSD plot is showing minor variations, TLR2 structure is very stable and no global conformational changes were seen. Next, $R g$ estimation of the system was done. $\mathrm{Rg}$ is a general feature of describing protein compactness and relaxation over simulation time. A higher variation of $R g$ plot implies less compactness and vice versa. The average $R g$ estimated for the system is $40.57 \AA$ (maximum of $42.06 \AA$ ) complementing the rmsd findings and affirming stable and compact behavior of the protein in the presence of the vaccine. Furthermore, a general overview of the protein residues fluctuation was concluded from the rmsf calculation. Residues of the TLR2 active residues key in binding vaccine and stabilizing are also elucidated in this assay. The average residue deviation is $3.9 \AA$ (maximum of $6.1 \AA$ ). This depicts highly stable behavior of the TLR2 residues after vaccine binding. Furthermore, the strength of intermolecular interactions was determined via hydrogen bonds analysis that demonstrated on average the formation of 37 hydrogen bonds in each frame of the simulation. This highly complies with the strong stability of TLR2-vaccine complex.

Binding free energy estimation. To affirm the affinity of the vaccine for TLR2, MMGB/PBSA was used as a post-simulation processing to calculate the different free energies of the complex. To achieve this objective, MMPBSA and its counterpart MMGBSA are more considered more precise compared to docking predictions and are less expensive than free energy perturbation. Both methods are efficient program as an end state free energy calculation method. The different binding free-energies calculated in MMGBSA and MMPBSA are presented in Tables 2 and 3 respectively. The analysis revealed a net delta energy of $-43.6643 \mathrm{kcal} / \mathrm{mol}$ in 
A

ATGACCCCGCAGAACATCACCGACCTGTGCGCTGAATACCACAACACCCAGATCCACACCCTGAACGACAA AATCTTCTCTTACACCGAATCTCTGGCTGGTAAACGTGAAATGGCTATCATCACCTTCAAAAACGGTGCTACC TTCCAGGTTGAAGTTCCGGGTTCTCAGCACATCGACTCTCAGAAAAAAGCTATCGAACGTATGAAAGACAC CCTGCGTATCGCTTACCTGACCGAAGCTAAAGTTGAAAAACTGTGCGTTTGGAACAACAAAACCCCGCACG CTATCGCTGCTATCTCTATGGCTAACGAAGCTGCTGCTAAACAGGCTGACGAAACCAACGCTACCATGGGTC CGGGTCCGGGTCTGACCGCTACCAACCCGGACGACGTTGGTCCGGGTCCGGGTTACTACGGTGGTCTGCAG TTCAACCTGGGTCCGGGTCCGGGTTCTCGTGAAGAACAGATCCGTGTTGCTGAAAACGTTCTGGCTCGTGG TCCGGGTCCGGGTGGTGGTGACTGGAACTGTTCAAAAACGCTACCGCTACCATGAAAGGTCCGGGTCCGGG TCTGTGGGCTATGGCTCCGGCTCTGGTTGCTGCTCCGATGGCTCTGGGTCCGGGTCCGGGTCTGCTGATCTTC GCTTTCCTGGGTGTTACCGCTGCTGTTGGTGTT

B

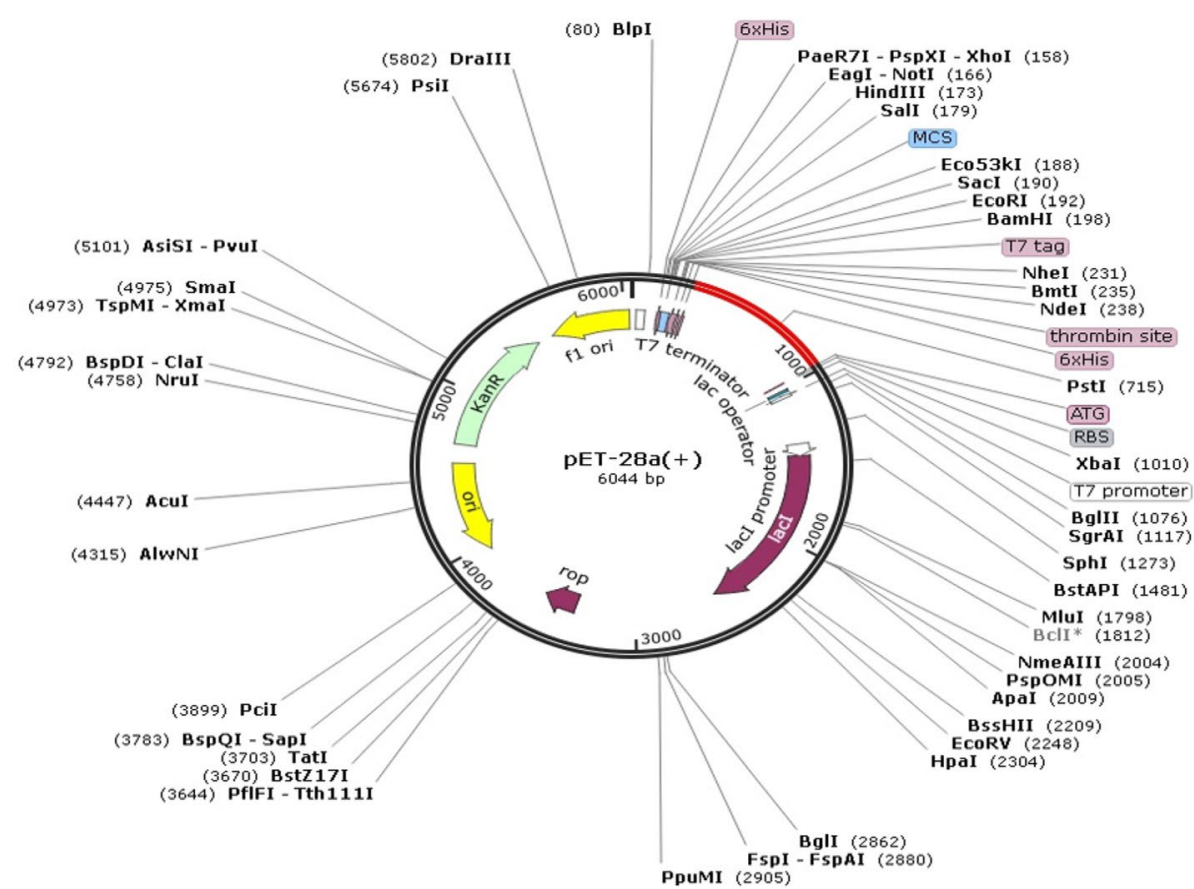

Figure 7. (A) The codon-optimized sequence of multi-epitope vaccine. (B) In silico cloning of polyepitope vaccine construct (shown in red) in pET28a expression vector.

MMGBSA and $-73.4728 \mathrm{kcal} / \mathrm{mol}$ in MMPBSA. In MMGBSA, delta energy revealed for the complex, TLR2, and vaccine is $-25,678.4794 \mathrm{kcal} / \mathrm{mol},-22,076.1979 \mathrm{kcal} / \mathrm{mol}$, and $-3558.6172 \mathrm{kcal} / \mathrm{mol}$, respectively. From the total MMPBSA energy, the vaccine binding energy is $-3451.8828 \mathrm{kcal} / \mathrm{mol}$. The complex binding energy is $-25,356.1860 \mathrm{kcal} / \mathrm{mol}$, TLR2 binding energy is $-21,830.8304 \mathrm{kcal} / \mathrm{mol}$. In both methods, the net electrostatic energy contributes favourably to the net binding energy. The net electrostatic energy of $-312.9003 \mathrm{kcal} /$ mol is estimated in both MMGBSA and MMPBSA for the system. The van der Waals energy is $-93.5882 \mathrm{kcal} /$ $\mathrm{mol}(\mathrm{complex}=-10,522.8197 \mathrm{kcal} / \mathrm{mol}, \mathrm{TLR} 2=-8786.4960 \mathrm{kcal} / \mathrm{mol}$, and vaccine $=-1642.7355 \mathrm{kcal} / \mathrm{mol})$. The net solvation free energy is revealed non-favourable to the total energy in MMGBSA $(301.5696 \mathrm{kcal} / \mathrm{mol})$ and MMPBSA $(271.7610 \mathrm{kcal} / \mathrm{mol})$.

\section{Discussion}

While M. abscessus infection may not represent an immediate threat, medical attention on this opportunistic pathogen is only growing with time, and rightfully so. There is still time and a window of opportunity to manage these bacterial infections by developing effective therapies such as vaccines before the emergence of more drug resistant strains of $M$. abscessus which is inevitably occurring due to the widespread and uncontrolled use of antibiotics.

The ratio of the core and pan-genome sizes was found to be 0.34 . It means that the core genome is approximately $34 \%$ of the pangenome. This indicates $M$. abscessus has an open pangenome which will continue to receive new genes. It is possible that horizontal gene transfer has a crucial role in the evolution of this emerging microorganism thus enabling it to acquire increasing genetic material. In our study, it was found that bacterial isolates from different areas of the world clustered together i.e., from the USA to Malaysia, Russia, and China. This observation indicates that the global dissemination of M. abscessus is ongoing. Exploring the phylogeny results 

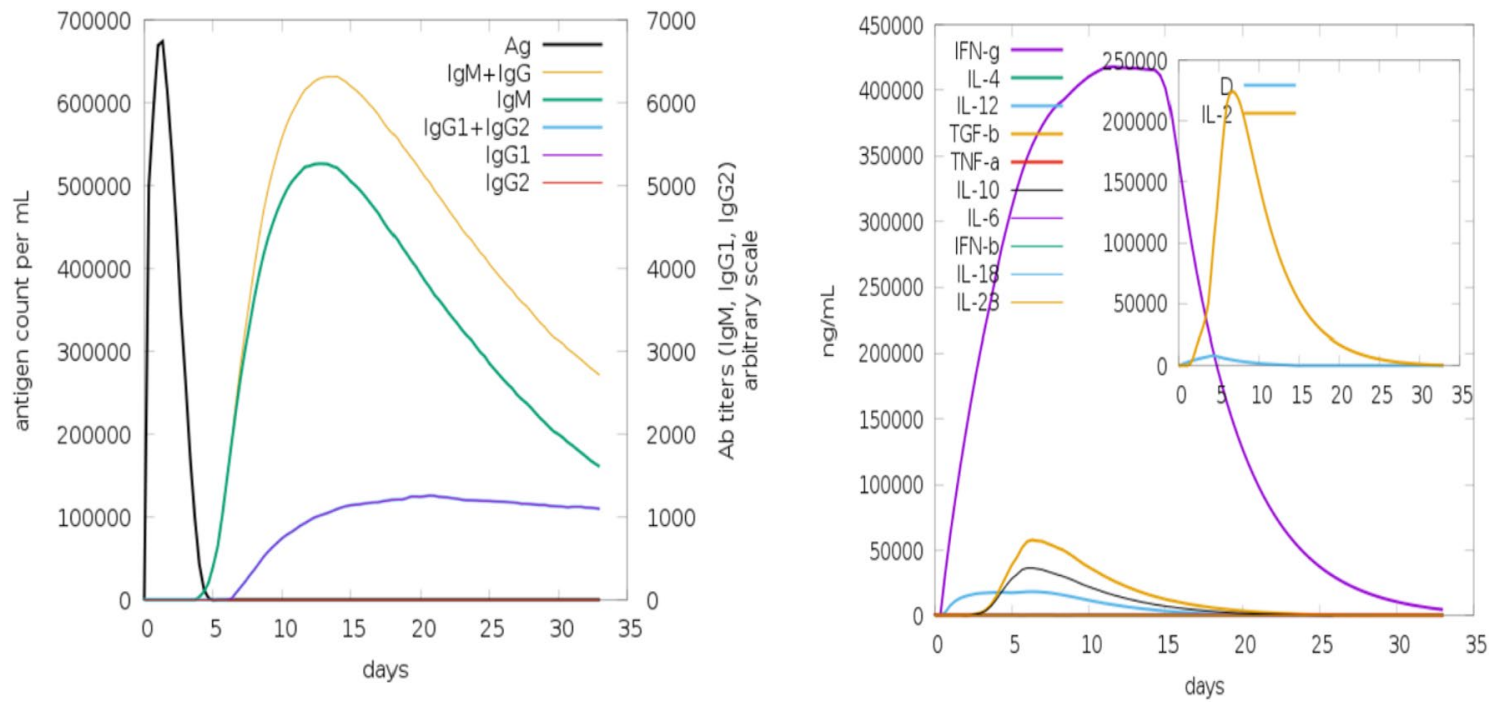

Figure 8. C-immune simulation to verify the host immune system's reaction against the polyepitope-based vaccine engineered. At the left there are antibodies, whereas cytokines and interleukins are shown in right.

A

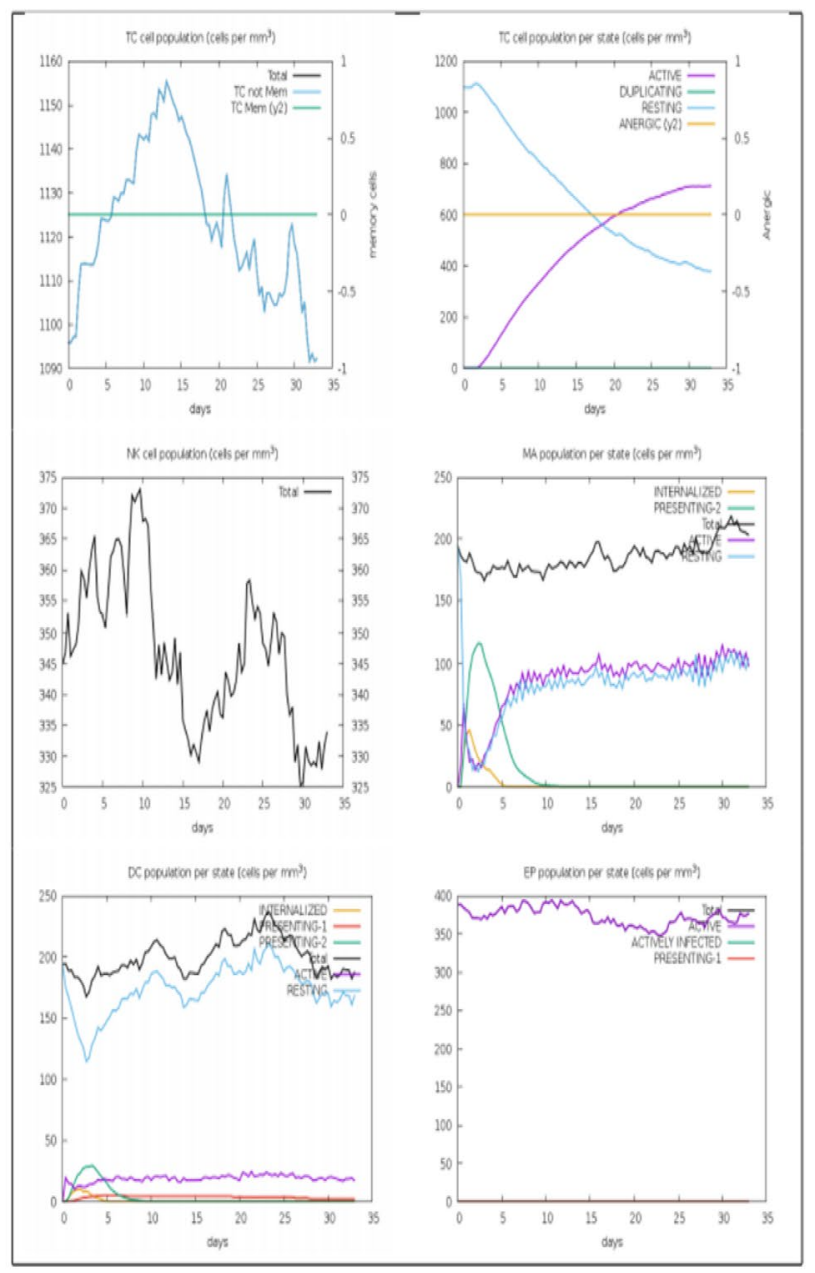

B
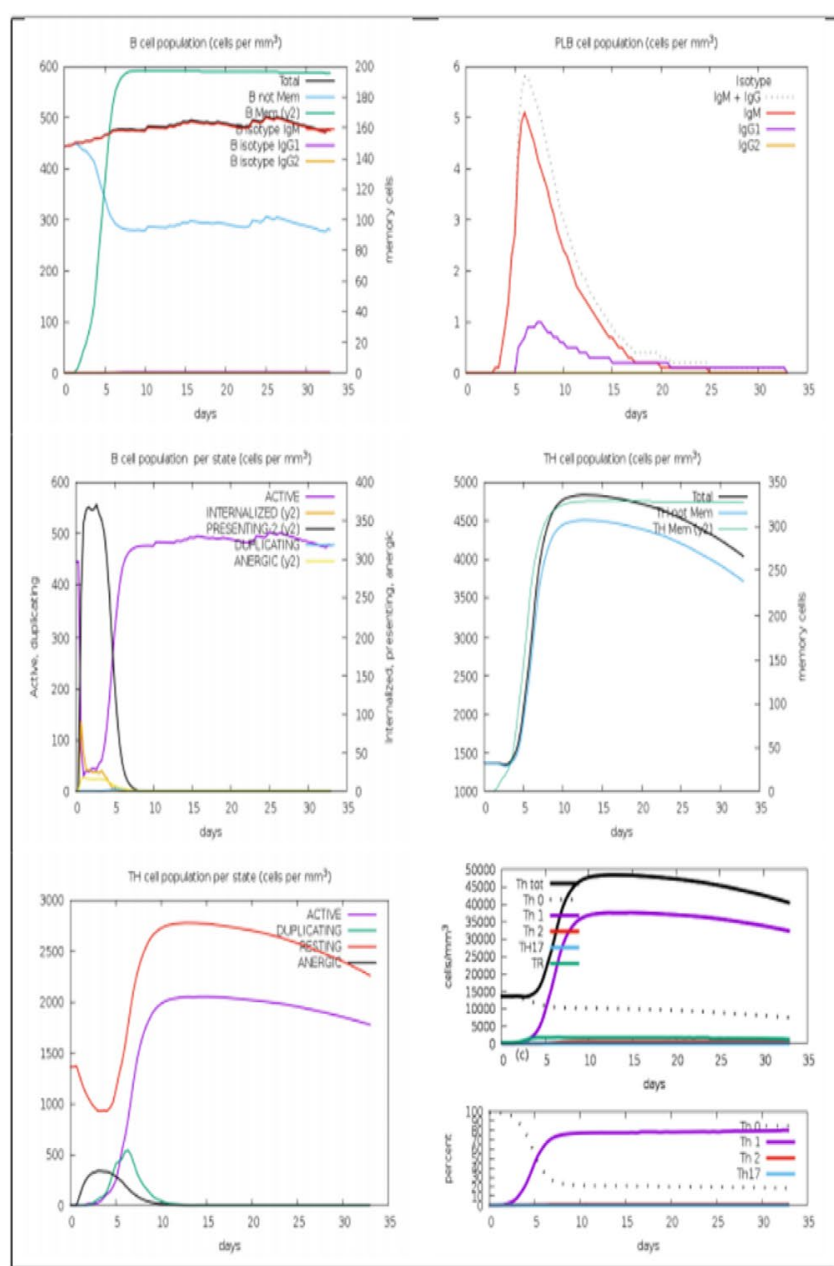

Figure 9. (A) Population of B and T cells versus days in response to polyepitope vaccine construct antigen. (B) Count of immune cells formed against the response of polyepitope vaccine construct; TC, NK, MA, DC and EP (Cytotoxic T cells, Natural killer cells, Macrophages, Dendritic cells and Epithelial cells). 

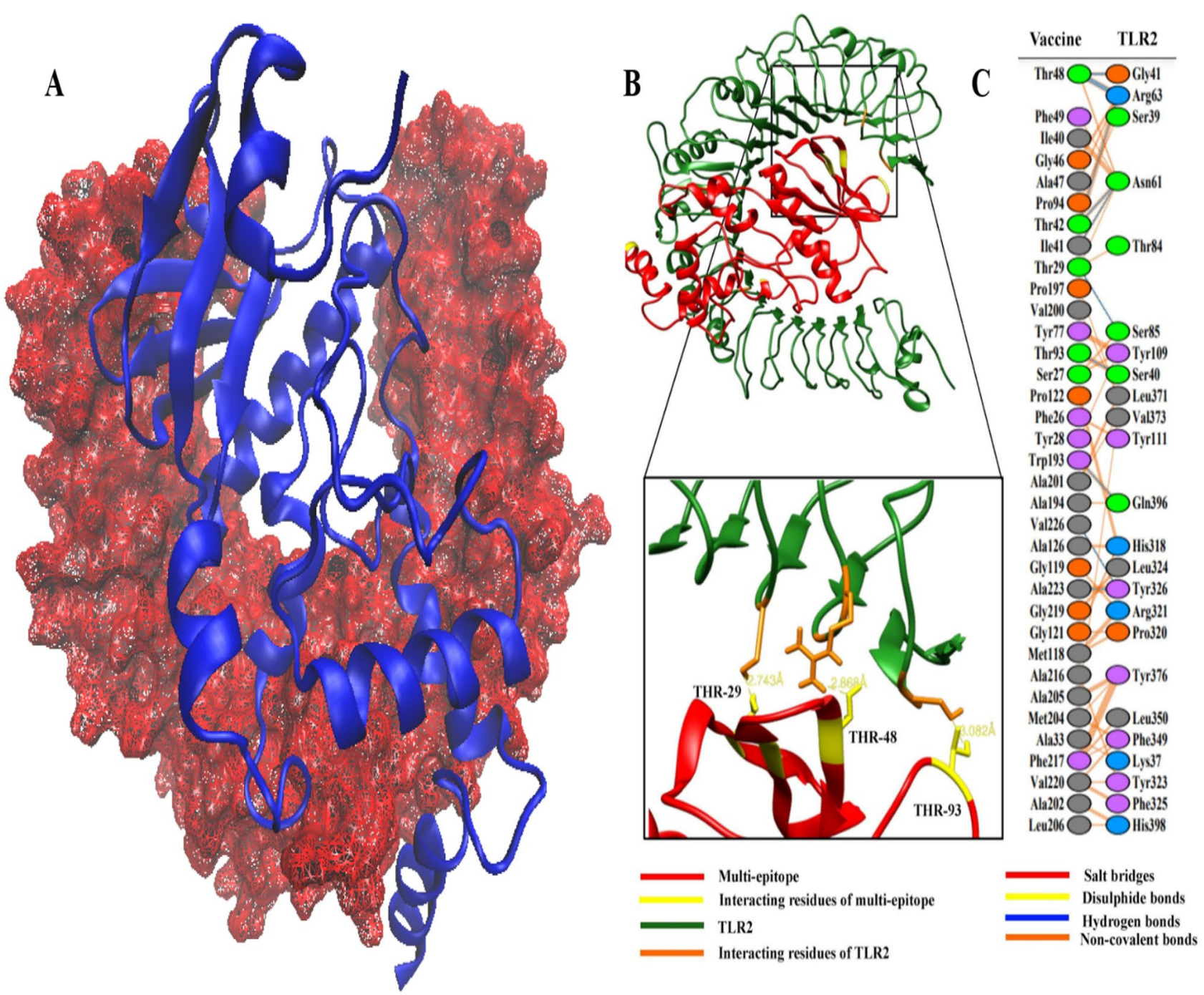

Figure 10. (A) Polyepitope vaccine construct (shown in NewCartoon Blue) conformation with respect to TLR2 receptor (shown in wireframe surf Red). (B) Analysis of refined vaccine-TLR2 complex at inter-chain level and residue. (C) Visualization of some notable inter-chain hydrogen bonds.

confirmed that pangenome phylogeny (Fig. 2) provided better resolution to discriminate between the bacterial strains vis-a-vis the core phylogeny. The pangenome based tree clearly separated closely related strains that were otherwise depicted phylogenetically closer in the core genome phylogeny. Apart from that, useful insights were drawn from the genomics data. The variation in genome size, GC content and protein coding genes within $M$. abscessus strains make clear physiological and genomic differences between them and the elaborated genetic makeup of the species in the natural environment/niche.

Apart from genome analysis of $M$. abscessus, we took an aim to design a multi-epitope vaccine against this opportunistic pathogen. A reverse vaccinology pipeline Vaxign was used to select exoproteome and secretomerelated proteins as targets localized in these regions have the increased tendency to act as antigens ${ }^{42}$. However, to overcome the limitation of this tool, more filters (antigenicity, physicochemical characterization, transmembrane helices less than two) were also considered to prioritize antigens for vaccine design.

Epitope mapping and selection was conducted to identify immunogenic regions within the four core antigenic proteins. It was found that the selected T-cell epitopes were conserved and antigenic so they might be useful components of a broad-spectrum vaccine. The prediction of discontinuous B-cell epitopes was carried out on the three-dimensional structure of multi-epitope vaccine to validate the presence of B-cell epitopes and remove false positive hits. There is a growing consensus that B-cell epitopes are mostly conformational in nature as opposed to being linear ${ }^{43-45}$. Thus, it was reasoned that sequence-based analysis is not suitable to identify potential B-cell epitopes. That is why an endeavor was made to first obtain the high quality and stabilized structural model of the polyepitope vaccine and then use it as input for programs like Ellipro and DISCOTOPE for 3D structurebased epitope prediction.

Encouraging results were obtained in case of conformational B-cell epitope mapping within the designed multi-epitope vaccine. This development indicated that besides activating T-cell response, our designed vaccine will serve to activate B-cells too and lead to the induction of specific humoral responses. Indeed, vaccines that activate both arms of adaptive immunity are likely to resolve microbial infections due to the emphasis on both 

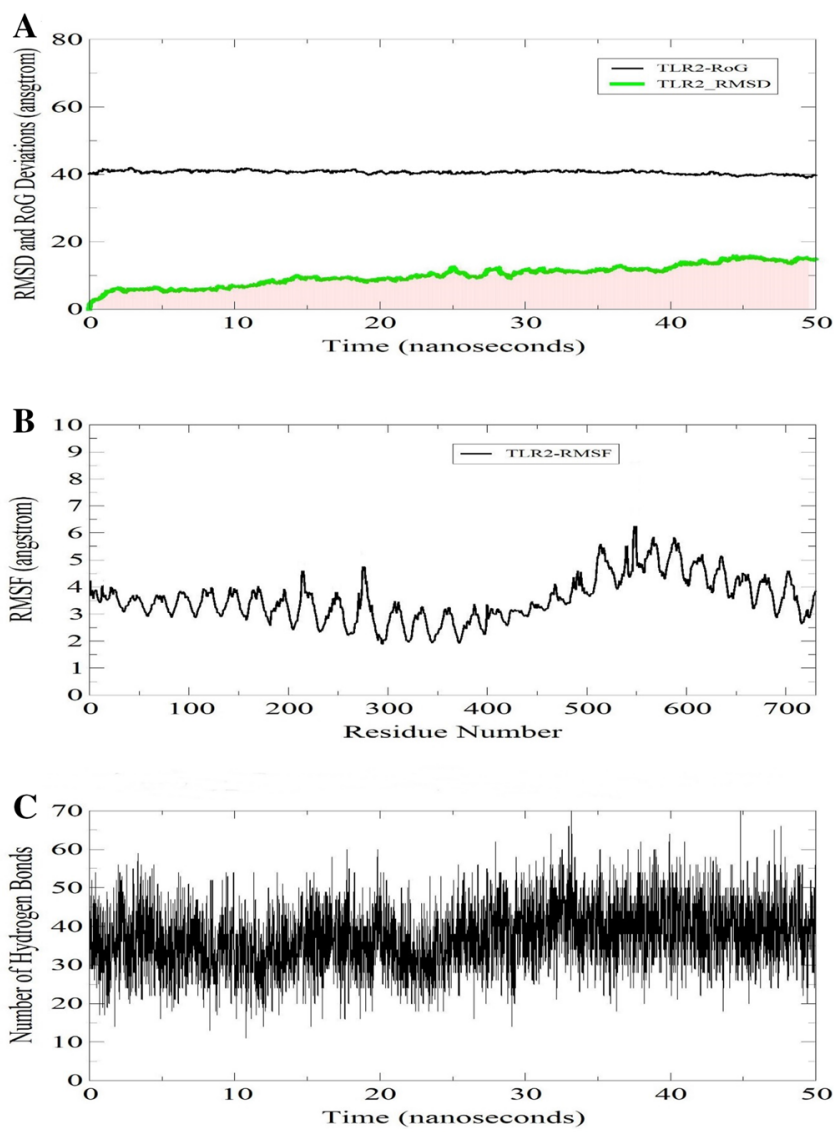

Figure 11. Molecular dynamic simulation of TLR2-vaccine complex. (A) Graph showing RMSD and RoG. (B) Graph showing fluctuations in RMSF during simulations. (C) Hydrogen bond analysis during simulations.

the cellular and humoral aspects of immunity ${ }^{46}$. However, the major focus of this study was on the activation of T-cell immunity. Vaccine-elicited T-cell responses, in addition to being durable, can circumvent the antigenic drift that in turn is linked to the evasion of antibody memory responses ${ }^{47}$.

The multi-epitope vaccine showed good physicochemical features in terms of thermodynamic feasibility, stability, hydrophilicity and expression capacity. The multi-epitope vaccine is non-allergen, thus harmful responses of the vaccine are not expected. The vaccine designed in the present study exhibited a high level of antigenicity which is much preferred for immunological applications. In addition, overexpressed of this vaccine could be done in Escherichia coli K12 strain.

Altogether, the predictive framework for multi-epitope design and its downstream analysis using molecular docking and interaction analysis is a good starting point for the development of vaccine candidates. However, further in vitro and in vivo studies are necessary to establish whether the epitopes and multi-epitope vaccine proposed in this study will confer protective immunity against M. abscessus infection or not.

\section{Conclusions}

M. abscessus is an emerging multi-drug resistant infectious pathogen and has the potential to exert serious infections in humans. Development of a vaccine that could protect humans from infections of the said pathogen would be a breakthrough. Due to the complex biology of the pathogen and the daunting nature of conventional vaccine development, vaccine development against this pathogen is challenging. Therefore, we herein formulate an in silico vaccine based on antigenic epitopes collected from across the pathogen's core antigenic proteins to develop an effective vaccine candidate. The selected epitopes were selected from the core genome thus enhancing the broad-spectrum of the designed vaccine ensemble. The vaccine has also demonstrated to elicit both major humoral and cellular immunity. Notably, the included epitopes in the vaccine construct are non-homologous to the human host, antigenic, and non-allergic. The vaccine ensemble itself possesses all good qualities needed for a practical vaccine candidate and is showing high affinity and stability of binding to TLR2 innate immune receptor, the interaction with which is vital in recognition and processing by the host immune system. The use of all these immunoinformatics and biophysical approaches are indeed extremely useful in guiding experimental studies and saving time and cost. However, because of the limitation of these used tools and servers, in vitro immunological assays are recommended to evaluate the biological protection efficacy of the vaccine. 


\begin{tabular}{|c|c|c|c|}
\hline Energy component & Average & SD & SE of mean \\
\hline \multicolumn{4}{|l|}{ Generalized born } \\
\hline \multicolumn{4}{|l|}{ Complex } \\
\hline Van der Waals & $-10,522.8197$ & 53.7628 & 5.3763 \\
\hline Electrostatic & $-95,157.9075$ & 174.7182 & 17.4718 \\
\hline Polar solvation energy & $-16,023.2839$ & 140.2591 & 14.0259 \\
\hline Non-polar solvation energy & 472.8864 & 4.4365 & 0.4437 \\
\hline Gas phase energy & $-10,128.0819$ & 196.1939 & 19.6194 \\
\hline Solvation energy & $-15,550.3975$ & 138.8135 & 13.8813 \\
\hline Total & $-25,678.4794$ & 148.6605 & 14.8661 \\
\hline \multicolumn{4}{|l|}{ TLR2 receptor } \\
\hline Van der Waals & -8786.4960 & 51.1912 & 5.1191 \\
\hline Electrostatic & $-77,040.1613$ & 169.8608 & 16.9861 \\
\hline Polar solvation energy & $-12,863.0115$ & 145.4925 & 14.5492 \\
\hline Non-polar solvation energy & 358.4210 & 3.9722 & 0.3972 \\
\hline Gas phase energy & -9571.6074 & 178.9572 & 17.8957 \\
\hline Solvation energy & $-12,504.5906$ & 144.2641 & 14.4264 \\
\hline Total & $-22,076.1979$ & 124.3022 & 12.4302 \\
\hline \multicolumn{4}{|l|}{ Vaccine } \\
\hline Van der Waals & -1642.7355 & 23.7365 & 2.3737 \\
\hline Electrostatic & $-17,804.8459$ & 97.0236 & 9.7024 \\
\hline Polar solvation energy & -3475.5293 & 81.4546 & 8.1455 \\
\hline Non-polar solvation energy & 128.1528 & 2.6121 & 0.2612 \\
\hline Gas phase energy & -211.2407 & 94.4941 & 9.4494 \\
\hline Solvation energy & -3347.3765 & 80.1900 & 8.0190 \\
\hline Total & -3558.6172 & 51.8689 & 5.1869 \\
\hline \multicolumn{4}{|c|}{ Differences (Complex-TLR2-Vaccine) } \\
\hline Van der Waals & -93.5882 & 9.9121 & 0.9912 \\
\hline Electrostatic & -312.9003 & 46.2789 & 4.6279 \\
\hline Polar solvation energy & 315.2570 & 43.5847 & 4.3585 \\
\hline Non-polar solvation energy & -13.6874 & 1.3925 & 0.1393 \\
\hline Gas phase energy & -345.2339 & 45.0664 & 4.5066 \\
\hline Solvation energy & 301.5696 & 43.2835 & 4.3284 \\
\hline Total & -43.6643 & 6.8491 & 0.6849 \\
\hline
\end{tabular}

Table 2. MMGBSA based binding free energies of the complex, TLR2, vaccine and net delta energy in kcal/ mol. 


\begin{tabular}{|c|c|c|c|}
\hline Energy component & Average & SD & SE of mean \\
\hline \multicolumn{4}{|l|}{ Poisson Boltzmann } \\
\hline \multicolumn{4}{|l|}{ Complex } \\
\hline Van der Waals & $-10,522.8197$ & 53.7628 & 5.3763 \\
\hline Electrostatic & $-95,157.9075$ & 174.7182 & 17.4718 \\
\hline Polar solvation energy & $-15,551.4301$ & 134.6322 & 13.4632 \\
\hline Non-polar solvation energy & 323.3260 & 1.7048 & 0.1705 \\
\hline Gas phase energy & $-10,128.0819$ & 196.1939 & 19.6194 \\
\hline Solvation energy & $-15,228.1041$ & 134.0287 & 13.4029 \\
\hline Total & $-25,356.1860$ & 145.5654 & 14.5565 \\
\hline \multicolumn{4}{|l|}{ TLR2 receptor } \\
\hline Van der Waals & -8786.4960 & 51.1912 & 5.1191 \\
\hline Electrostatic & $-77,040.1613$ & 169.8608 & 16.9861 \\
\hline Polar solvation energy & $-12,507.7310$ & 140.3346 & 14.0335 \\
\hline Non-polar solvation energy & 248.5080 & 1.1626 & 0.1163 \\
\hline Gas phase energy & -9571.6074 & 178.9572 & 17.8957 \\
\hline Solvation energy & $-12,259.2230$ & 139.9714 & 13.9971 \\
\hline Total & $-21,830.8304$ & 123.7853 & 12.3785 \\
\hline \multicolumn{4}{|l|}{ Vaccine molecule } \\
\hline Van der Waals & -1642.7355 & 23.7365 & 2.3737 \\
\hline Electrostatic & $-17,804.8459$ & 97.0236 & 9.7024 \\
\hline Polar solvation energy & -3326.9413 & 82.7583 & 8.2758 \\
\hline Non-polar solvation energy & 86.2992 & 1.6065 & 0.1607 \\
\hline Gas phase energy & -211.2407 & 94.4941 & 9.4494 \\
\hline Solvation energy & -3240.6421 & 81.9779 & 8.1978 \\
\hline Total & -3451.8828 & 54.2440 & 5.4244 \\
\hline \multicolumn{4}{|c|}{ Differences (Complex-TLR2-Vaccine) } \\
\hline Van der Waals & -93.5882 & 9.9121 & 0.9912 \\
\hline Electrostatic & -312.9003 & 46.2789 & 4.6279 \\
\hline Polar solvation energy & 283.2422 & 44.5670 & 4.4567 \\
\hline Non-polar solvation Energy & -11.4811 & 1.2082 & 0.1208 \\
\hline Gas phase energy & -345.2339 & 45.0664 & 4.5066 \\
\hline Solvation energy & 271.7610 & 44.3420 & 4.4342 \\
\hline Total & -73.4728 & 9.1691 & 0.9169 \\
\hline
\end{tabular}

Table 3. MMPBSA based binding free energies of the complex, TLR2, vaccine and net delta energy in kcal/ mol.

Received: 11 February 2021; Accepted: 19 May 2021

Published online: 27 May 2021

\section{References}

1. Koh, S. J. et al. An outbreak of skin and soft tissue infection caused by Mycobacterium abscessus following acupuncture. Clin. Microbiol. Infect. 16, 895-901. https://doi.org/10.1111/j.1469-0691.2009.03026.x (2010).

2. Lee, M. R. et al. Mycobacterium abscessus complex infections in humans. Emerg. Infect. Dis. 21, 1638-1646. https://doi.org/10. 3201/2109.141634 (2015).

3. Gupta, R. S., Lo, B. \& Son, J. Phylogenomics and comparative genomic studies robustly support division of the genus Mycobacterium into an emended genus Mycobacterium and four novel genera. Front. Microbiol. 9, 67. https://doi.org/10.3389/fmicb.2018.00067 (2018).

4. Satyam, R., Bhardwaj, T., Jha, N. K., Jha, S. K. \& Nand, P. Toward a chimeric vaccine against multiple isolates of Mycobacteroides-an integrative approach. Life Sci. 250, 117541. https://doi.org/10.1016/j.lfs.2020.117541 (2020).

5. Li, B. et al. Genetic evolution of Mycobacterium abscessus conferring clarithromycin resistance during long-term antibiotic therapy. Can. Respir. J. 2020, 7623828. https://doi.org/10.1155/2020/7623828 (2020).

6. Degiacomi, G. et al. Mycobacterium abscessus, an emerging and worrisome pathogen among cystic fibrosis patients. Int. J. Mol. Sci. https://doi.org/10.3390/ijms20235868 (2019).

7. Roux, A. L. et al. The distinct fate of smooth and rough Mycobacterium abscessus variants inside macrophages. Open Biol. https:// doi.org/10.1098/rsob.160185 (2016).

8. Capecchi, B., Serruto, D., Adu-Bobie, J., Rappuoli, R. \& Pizza, M. The genome revolution in vaccine research. Curr. Issues Mol. Biol. 6, 17-27 (2004).

9. Dar, H. A. et al. Immunoinformatics-aided design and evaluation of a potential multi-epitope vaccine against Klebsiella pneumoniae. Vaccines (Basel) https://doi.org/10.3390/vaccines7030088 (2019).

10. Chaudhari, N. M., Gupta, V. K. \& Dutta, C. BPGA- an ultra-fast pan-genome analysis pipeline. Sci. Rep. 6, 24373. https://doi.org/ 10.1038/srep24373 (2016). 
11. Edgar, R. C. Search and clustering orders of magnitude faster than BLAST. Bioinformatics 26, 2460-2461. https://doi.org/10.1093/ bioinformatics/btq461 (2010).

12. He, Y., Xiang, Z. \& Mobley, H. L. Vaxign: the first web-based vaccine design program for reverse vaccinology and applications for vaccine development. J. Biomed. Biotechnol. 2010, 297505. https://doi.org/10.1155/2010/297505 (2010).

13. Barh, D. et al. Exoproteome and secretome derived broad spectrum novel drug and vaccine candidates in Vibrio cholerae targeted by Piper betel derived compounds. PLoS ONE 8, e52773. https://doi.org/10.1371/journal.pone.0052773 (2013).

14. Cusick, M. F., Libbey, J. E. \& Fujinami, R. S. Molecular mimicry as a mechanism of autoimmune disease. Clin. Rev. Allergy Immunol. 42, 102-111. https://doi.org/10.1007/s12016-011-8294-7 (2012).

15. Kline, K. A., Falker, S., Dahlberg, S., Normark, S. \& Henriques-Normark, B. Bacterial adhesins in host-microbe interactions. Cell Host Microbe 5, 580-592. https://doi.org/10.1016/j.chom.2009.05.011 (2009).

16. Wizemann, T. M., Adamou, J. E. \& Langermann, S. Adhesins as targets for vaccine development. Emerg. Infect. Dis. 5, 395-403. https://doi.org/10.3201/eid0503.990310 (1999).

17. Doytchinova, I. A. \& Flower, D. R. VaxiJen: a server for prediction of protective antigens, tumour antigens and subunit vaccines. BMC Bioinform. 8, 4. https://doi.org/10.1186/1471-2105-8-4 (2007).

18. Gasteiger, E. et al. ExPASy: The proteomics server for in-depth protein knowledge and analysis. Nucleic Acids Res. 31, 3784-3788. https://doi.org/10.1093/nar/gkg563 (2003).

19. Baseer, S., Ahmad, S., Ranaghan, K. E. \& Azam, S. S. Towards a peptide-based vaccine against Shigella sonnei: a subtractive reverse vaccinology based approach. Biologicals 50, 87-99. https://doi.org/10.1016/j.biologicals.2017.08.004 (2017).

20. Solanki, V. \& Tiwari, V. Subtractive proteomics to identify novel drug targets and reverse vaccinology for the development of chimeric vaccine against Acinetobacter baumannii. Sci. Rep. 8, 9044. https://doi.org/10.1038/s41598-018-26689-7 (2018).

21. Calis, J. J. et al. Properties of MHC class I presented peptides that enhance immunogenicity. PLoS Comput. Biol. 9, e1003266. https://doi.org/10.1371/journal.pcbi.1003266 (2013).

22. Fleri, W. et al. The immune epitope database and analysis resource in epitope discovery and synthetic vaccine design. Front. Immunol. 8, 278. https://doi.org/10.3389/fimmu.2017.00278 (2017).

23. Dimitrov, I., Naneva, L., Doytchinova, I. \& Bangov, I. AllergenFP: allergenicity prediction by descriptor fingerprints. Bioinformatics 30, 846-851. https://doi.org/10.1093/bioinformatics/btt619 (2014).

24. Cheng, J., Randall, A. Z., Sweredoski, M. J. \& Baldi, P. SCRATCH: a protein structure and structural feature prediction server. Nucleic Acids Res. 33, W72-76. https://doi.org/10.1093/nar/gki396 (2005).

25. Lee, G. R., Won, J., Heo, L. \& Seok, C. GalaxyRefine2: simultaneous refinement of inaccurate local regions and overall protein structure. Nucleic Acids Res. 47, W451-W455. https://doi.org/10.1093/nar/gkz288 (2019).

26. Laskowski, R. A., Rullmannn, J. A., MacArthur, M. W., Kaptein, R. \& Thornton, J. M. AQUA and PROCHECK-NMR: programs for checking the quality of protein structures solved by NMR. J. Biomol. NMR 8, 477-486. https://doi.org/10.1007/BF00228148 (1996).

27. Wiederstein, M. \& Sippl, M. J. ProSA-web: interactive web service for the recognition of errors in three-dimensional structures of proteins. Nucleic Acids Res. 35, W407-410. https://doi.org/10.1093/nar/gkm290 (2007).

28. Brubaker, S. W., Bonham, K. S., Zanoni, I. \& Kagan, J. C. Innate immune pattern recognition: a cell biological perspective. Annu. Rev. Immunol. 33, 257-290. https://doi.org/10.1146/annurev-immunol-032414-112240 (2015).

29. Sampaio, E. P. et al. Mycobacterium abscessus and M. avium trigger Toll-like receptor 2 and distinct cytokine response in human cells. Am. J. Respir. Cell Mol. Biol. 39, 431-439. https://doi.org/10.1165/rcmb.2007-0413OC (2008).

30. Jin, M. S. et al. Crystal structure of the TLR1-TLR2 heterodimer induced by binding of a tri-acylated lipopeptide. Cell 130, 1071-1082. https://doi.org/10.1016/j.cell.2007.09.008 (2007).

31. van Zundert, G. C. P. et al. The HADDOCK2.2 web server: user-friendly integrative modeling of biomolecular complexes. J. Mol. Biol. 428, 720-725. https://doi.org/10.1016/j.jmb.2015.09.014 (2016).

32. de Vries, S. J. \& Bonvin, A. M. CPORT: a consensus interface predictor and its performance in prediction-driven docking with HADDOCK. PLoS ONE 6, e17695. https://doi.org/10.1371/journal.pone.0017695 (2011).

33. Pettersen, E. F. et al. UCSF Chimera-a visualization system for exploratory research and analysis. J. Comput. Chem. 25, 1605-1612. https://doi.org/10.1002/jcc.20084 (2004).

34. Humphrey, W., Dalke, A. \& Schulten, K. VMD: visual molecular dynamics. J. Mol. Graph 14(33-38), 27-38. https://doi.org/10. 1016/0263-7855(96)00018-5 (1996).

35. Laskowski, R. A. PDBsum: summaries and analyses of PDB structures. Nucleic Acids Res. 29, 221-222. https://doi.org/10.1093/ nar/29.1.221 (2001).

36. Xue, L. C., Rodrigues, J. P., Kastritis, P. L., Bonvin, A. M. \& Vangone, A. PRODIGY: a web server for predicting the binding affinity of protein-protein complexes. Bioinformatics 32, 3676-3678. https://doi.org/10.1093/bioinformatics/btw514 (2016).

37. Case, D. A. et al. The Amber biomolecular simulation programs. J. Comput. Chem. 26, 1668-1688. https://doi.org/10.1002/jcc. 20290 (2005).

38. Maier, J. A. et al. ff14SB: improving the accuracy of protein side chain and backbone parameters from ff99SB. J. Chem. Theory Comput. 11, 3696-3713. https://doi.org/10.1021/acs.jctc.5b00255 (2015).

39. Wu, X., Brooks, B. R. \& Vanden-Eijnden, E. Self-guided Langevin dynamics via generalized Langevin equation. J. Comput. Chem. 37, 595-601. https://doi.org/10.1002/jcc.24015 (2016).

40. Roe, D. R. \& Cheatham, T. E. 3rd. PTRAJ and CPPTRAJ: software for processing and analysis of molecular dynamics trajectory data. J. Chem. Theory Comput. 9, 3084-3095. https://doi.org/10.1021/ct400341p (2013).

41. Miller, B. R. 3rd. et al. MMPBSA.py: an efficient program for end-state free energy calculations. J. Chem. Theory Comput. 8, 3314-3321. https://doi.org/10.1021/ct300418h (2012).

42. Naz, A. et al. Identification of putative vaccine candidates against Helicobacter pylori exploiting exoproteome and secretome: a reverse vaccinology based approach. Infect. Genet. Evol. 32, 280-291. https://doi.org/10.1016/j.meegid.2015.03.027 (2015).

43. Corcoran, A., Mahon, B. P. \& Doyle, S. B cell memory is directed toward conformational epitopes of parvovirus B19 capsid proteins and the unique region of VP1. J. Infect. Dis. 189, 1873-1880. https://doi.org/10.1086/382963 (2004).

44. Kozlova, E. E. G. et al. Computational B-cell epitope identification and production of neutralizing murine antibodies against Atroxlysin-I. Sci. Rep. 8, 14904. https://doi.org/10.1038/s41598-018-33298-x (2018).

45. Potocnakova, L., Bhide, M. \& Pulzova, L. B. An introduction to B-cell epitope mapping and in silico epitope prediction. J. Immunol. Res. 2016, 6760830. https://doi.org/10.1155/2016/6760830 (2016).

46. Clem, A. S. Fundamentals of vaccine immunology. J. Glob. Infect. Dis. 3, 73-78. https://doi.org/10.4103/0974-777X.77299 (2011).

47. Bai, X. F., Liu, J., Li, O., Zheng, P. \& Liu, Y. Antigenic drift as a mechanism for tumor evasion of destruction by cytolytic T lymphocytes. J. Clin. Investig. 111, 1487-1496. https://doi.org/10.1172/JCI17656 (2003).

\section{Acknowledgements}

We would like to acknowledge the administrative support provided by Foundation University Medical College, Foundation University, Pakistan. K.M.s work is supported by United Arab Emirates University Start up grant\#G00003347 and UAEU-UPAR-Grant\#G00003458. 


\section{Author contributions}

H.A.D.: Formal analysis, methodology, software, visualization; Y.W.: Data curation, project administration, resources, supervision; S.I.: writing-original draft preparation, software, conceptualization; S.A.: writing-original draft preparation, investigation; Z.J.: writing-reviewing and editing; H.A.: formal analysis, writing and editing. H.F.H.: visualization, formal analysis; K.M.: validation, methodology, and supervision.

\section{Funding}

This research did not receive any specific grant from funding agencies in the public, commercial, or not-forprofit sectors.

\section{Competing interests}

The authors declare no competing interests.

\section{Additional information}

Supplementary Information The online version contains supplementary material available at https://doi.org/ 10.1038/s41598-021-90868-2.

Correspondence and requests for materials should be addressed to Y.W. or K.M.

Reprints and permissions information is available at www.nature.com/reprints.

Publisher's note Springer Nature remains neutral with regard to jurisdictional claims in published maps and institutional affiliations.

(c) (i) Open Access This article is licensed under a Creative Commons Attribution 4.0 International

License, which permits use, sharing, adaptation, distribution and reproduction in any medium or format, as long as you give appropriate credit to the original author(s) and the source, provide a link to the Creative Commons licence, and indicate if changes were made. The images or other third party material in this article are included in the article's Creative Commons licence, unless indicated otherwise in a credit line to the material. If material is not included in the article's Creative Commons licence and your intended use is not permitted by statutory regulation or exceeds the permitted use, you will need to obtain permission directly from the copyright holder. To view a copy of this licence, visit http://creativecommons.org/licenses/by/4.0/.

(C) The Author(s) 2021 